\title{
Ro-inversional Spectrum of Ammonia
}

\author{
G. Winnewisser ${ }^{\mathrm{a}}$, S. P. Belov ${ }^{\mathrm{a}, *}$, Th. Klaus ${ }^{\mathrm{a}}$, and $\check{S}_{\text {. Urban }}^{\mathrm{b}}$ \\ ${ }^{a}$ I. Physikalisches Institut, Universität zu Köln, D-50937 Köln. \\ b J. Heyrovský Institute of Physical Chemistry, Dolejškova 3, CZ-182 23 Praha 8
}

\section{Z. Naturforsch. 51 a, 200-206 (1996); received February 24, 1996}

The ground state $J=2-1, K=0$ and $K=1$ ro-inversional spectrum of ${ }^{14} \mathrm{NH}_{3}$ and ${ }^{15} \mathrm{NH}_{3}$ at $1.2 \mathrm{THz}$ has been measured with an accuracy of $20 \mathrm{kHz}$ using the Cologne terahertz spectrometer. The measured frequencies for the $K=0$ components are:

$$
\begin{array}{ll}
{ }^{14} \mathrm{NH}_{3} \quad(J, K)=a(2,0)-s(1,0): & 1214852.942(20) \mathrm{MHz}, \\
{ }^{15} \mathrm{NH}_{3} \quad(J, K)=a(2,0)-s(1,0): & 1210889.556(20) \mathrm{MHz} .
\end{array}
$$

In addition we have determined from saturation dip measurements of the $J=1-0$ transition the spin-rotation constant $C_{\mathrm{N}}=6.7(3) \mathrm{kHz}$ and the unsplit line center frequency:

$$
{ }^{14} \mathrm{NH}_{3}(J, K)=s(1,0)-a(0,0): 572498.163(10) \mathrm{MHz} .
$$

The new results are in excellent agreement with existing high resolution Fourier transform data. The terahertz line frequencies are of considerably higher accuracy than the FT-data, by about two orders of magnitude. They will serve as future calibration lines. The ro-inversional transitions are of astrophysical interest.

\section{Introduction}

In the development of spectroscopy the symmetric top molecule ammonia, $\mathrm{NH}_{3}$, has always played an important role from an experimental, theoretical and astrophysical point of view, and it continues to do so. It's pyramidal structure leads to the well known inversional transitions in the $1.2 \mathrm{~cm}$ wave region, which were the first molecular lines to be measured in absorption by microwave techniques [1]. These inversional transitions were used by Townes and coworkers to demonstrate in molecular beams the principle of state selection and thus maser action [2]. The very same transitions gave the first evidence of the existence of polyatomic molecules in interstellar space [3].

The special characteristics of ammonia are partly due to the fact that no other pyramidal symmetric

\footnotetext{
* On leave of absence from the Microwave Spectroscopy Laboratory, Institute of Applied Physics, Nizhnii Novgorod, Russia 603204

Reprint requests to Prof. Dr. G. Winnewisser.
}

top molecule exhibits inversion transitions which fall into the microwave region. In addition, several important properties distinguish $\mathrm{NH}_{3}$ from other symmetric top molecules: the existence of metastable and nonmetastable states, ortho- and para- modifications, hyperfine structure, and the occurrence of pure inversion and ro-inversion transitions. Theoretically, the inversional spectrum helped to understand the double minima potential problem [4], [5]. Since this early work, a vast amount of experimental and theoretical work has been performed on $\mathrm{NH}_{3}$, and in consequence it is well understood in the laboratory [6], [7]. Although the pure inversion transitions arise from levels of greatly different excitation energy, and thus are sensitive to a wide range of astrophysical excitation conditions, they fortuitously fall in the narrow frequency range $20-40 \mathrm{GHz}$, easily accessible to radio telescopes.

Since $\mathrm{NH}_{3}$ is a fairly light hydride molecule, the lowest ground state ro-inversion $\mathrm{R}$ branch transitions commence in the submillimeter wave region at 0.52 $\mathrm{mm}$ for $J=1-0$ and extend with increasing $J$ very rapidly into the far infrared region. The spacing is about $595 \mathrm{GHz}$ but modified by the effect of inversion. With microwave techniques it is very difficult 
to measure the ro-inversion transitions. In fact, their "microwave" recording poses a formidable technical problem because of the notoriously limited power output of the available radiation sources. Consequently, the first laboratory measurement of the $J=1-0$ transition was not carried out until 1967, when Helminger and Gordy [8], [9] used frequency multiplication techniques for recording this line. The very same transition was also observed by the Nizhnii Novgorod laboratory, employing the fundamental output radiation from a high frequency backward wave oscillator, BWO [10]. Although the ro-inversional spectrum has been recorded precisely by high-resolution Fourier-transform spectroscopy and has been analysed in great detail [11], [12], the measurement of the ro-inversion transitions by Doppler limited or even sub-Doppler spectroscopy remains a rewarding challenge. Aside from the considerably higher precision of the line center determination reached by Doppler limited spectroscopy, the finer details such as the hyperfine structure of the spectra are revealed by subDoppler techniques. The Doppler limited resolution of terahertz spectroscopy provides absolute line positions within $\pm 5 \mathrm{kHz}$ for unblended, strong lines [13], and are thus valuable wavelength calibrators for the far IR region, where the resolution of the FT spectra is generally instrument limited.

In fact, because of technical difficulties, which are mainly due to the lack of adequate radiation sources, the second ro-inversion transition $J=2-1$, which occurs in the terahertz region, has not been measured with microwave techniques until now. It is the purpose of the present paper to report its first successful measurement at 1.2 THz. At the same time, we report improved Lamb-dip spectra for the $J=1-0$ transition. Astrophysically, the ro-inversion transitions probe highdensity and very localized gas. The transitions of the $K=0$ ladder are of particular astrophysial importance, since nuclear spin statistics prevents the occurrence of pure inversion lines and thus the $K=0$ lines can only be observed via their ro-inversion transitions.

\section{Terahertz spectra}

\section{Experimental details}

The Cologne terahertz spectrometer has been described earlier in some detail [14] and it is operated at present with essentially two power sources. The
Table 1. Comparison between observed and calculated frequencies for $\mathrm{NH}_{3}$.

\begin{tabular}{|c|c|c|c|c|}
\hline$i^{\prime}\left(J^{\prime}, K^{\prime}\right.$ & $-i^{\prime \prime}\left(J^{\prime \prime}, K\right.$ & $\begin{array}{c}\nu_{\exp }(\mathrm{submm})^{\mathrm{a})} \\
/ \mathrm{MHz}\end{array}$ & $\begin{array}{c}\Delta \nu_{\exp }^{\text {b) }} \\
/ \mathrm{MHz}\end{array}$ & $\begin{array}{c}\Delta \nu_{\exp }{ }^{\mathrm{c})} \\
/ \mathrm{MHz}\end{array}$ \\
\hline \multicolumn{5}{|l|}{${ }^{14} \mathrm{NH}_{3}:$} \\
\hline$s(2,1)$ & $-a(1,1)$ & $1168452.394(20)$ & & 0.244 \\
\hline$a(2,0)$ & $-s(1,0)$ & $1214852.942(20)$ & & 0.402 \\
\hline$a(2,1)$ & $\leftarrow s(1,1)$ & $1215245.714(20)$ & & 0.354 \\
\hline \multicolumn{5}{|l|}{${ }^{15} \mathrm{NH}_{3}:$} \\
\hline$s(2,1)$ & $\leftarrow a(1,1)$ & $1166608.022(20)$ & $1.287(1.8)$ & -0.302 \\
\hline$a(2,0)$ & $\leftarrow s(1,0)$ & $1210889.556(20)$ & $-1.992(1.2)$ & -0.362 \\
\hline$a(2,1)$ & $-s(1,1)$ & $1211277.236(20)$ & $-0.524(1.2)$ & -0.314 \\
\hline
\end{tabular}

a) The estimated experimental uncertainty is given in brackets; ${ }^{\text {b) }}$ Differences between the experimental line positions measured by the Cologne terahertz spectrometer and the FT spectrometer at Gießen [17]. In brackets the experimental uncertainties from [17] are given in $\mathrm{MHz} ;{ }^{\mathrm{c})}$ Differences of $\nu_{\text {exp }}$ and calculated frequencies based on previous MW and FIR data of ${ }^{14} \mathrm{NH}_{3}$. For ${ }^{15} \mathrm{NH}_{3}$ the calculations were taken from [17].

frequency coverage is achieved either (i) by employing widely tunable BWOs as fundamental radiation sources [14] or (ii) by the use of frequency multipliers driven by BWOs $[15,16]$. The frequency output of the BWOs is frequency and phase locked via a millimeter wave synthesizer to a rubidium standard, which served as an atomic clock with a long term frequency stability of $10^{-11} \mathrm{sec}$. The new $\mathrm{NH}_{3}$ spectra were recorded with the Cologne terahertz spectrometer by operating a $1.2 \mathrm{THz}$ BWO (OB-83) for measurement of the $J=2-1$ spectrum and a second BWO (OB-80) to obtain the $J=1-0$ saturation spectra. The ammonia spectra were recorded using a $4 \mathrm{~m}$ long absorption cell in a flow system with Ar as a buffer gas to ensure low pressures of about $4 \mu \mathrm{bar}$ in order to avoid the spectra from turning optically thick.

\section{$J=2-1$ spectrum}

Up till now, the $J=2-1$ ro-inversion transition has been recorded in the laboratory only by Fouriertransform techniques. We report for the first time Doppler-limited measurements of the $(J, K)=a(2$, $0)-s(1,0)$, and the inversion split $K=1$ components $s(2,1)-a(1,1)$, and $a(2,1)-s(1,1)$ for ${ }^{14} \mathrm{NH}_{3}$ and ${ }^{15} \mathrm{NH}_{3}$, the latter in natural abundance. The new measurements are summarized in Table 1, together with the calculated frequencies. The recent FT measurements by Urban et al. [17] and their calculated 


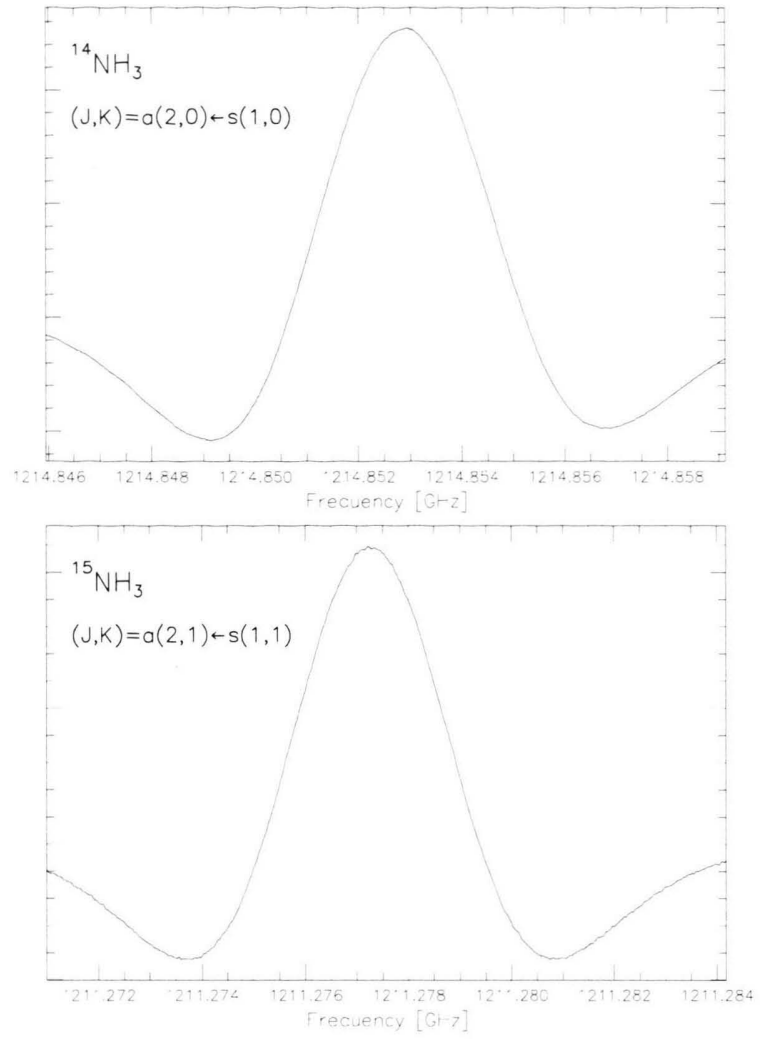

Fig. 1. Recording of two $J=2-1$ transitions of ammonia. The lines were recorded in second derivative form. ${ }^{15} \mathrm{NH}_{3}$ was observed in natural abundance, i. e. $0.366 \%$.

frequencies of the ${ }^{15} \mathrm{NH}_{3}$ ro-inversional transitions agree to within $400 \mathrm{kHz}$ with our present numbers. This close agreement confirms the high degree of accuracy and reliability of the FT measurements and furnishes evidence that the least squares analysis to the standard effective Hamiltonian for symmetric tops in the ground state is highly trustworthy. It might be noted that the calculated wavenumbers quoted in Table 1 of [17] by Urban et al. are obtained from the Padé approximant, but the same quality of fit is achieved by employing the conventional polynomial approximation. The calculated frequencies of the roinversional transitions quoted in Table 1 for comparison with the newly measured transitions are based on a comprehensive analysis using a sophisticated effective Hamiltonian, taking into account the matrix elements up to decadic order, including all the corresponding off-diagonal matrix elements [12].

Figure 1 presents two recorded $J=2-1$ lines in order to give an impression of the measured line profiles
${ }^{14} \mathrm{NH}_{3}$ : Energy level diagram

$\mathrm{J}$

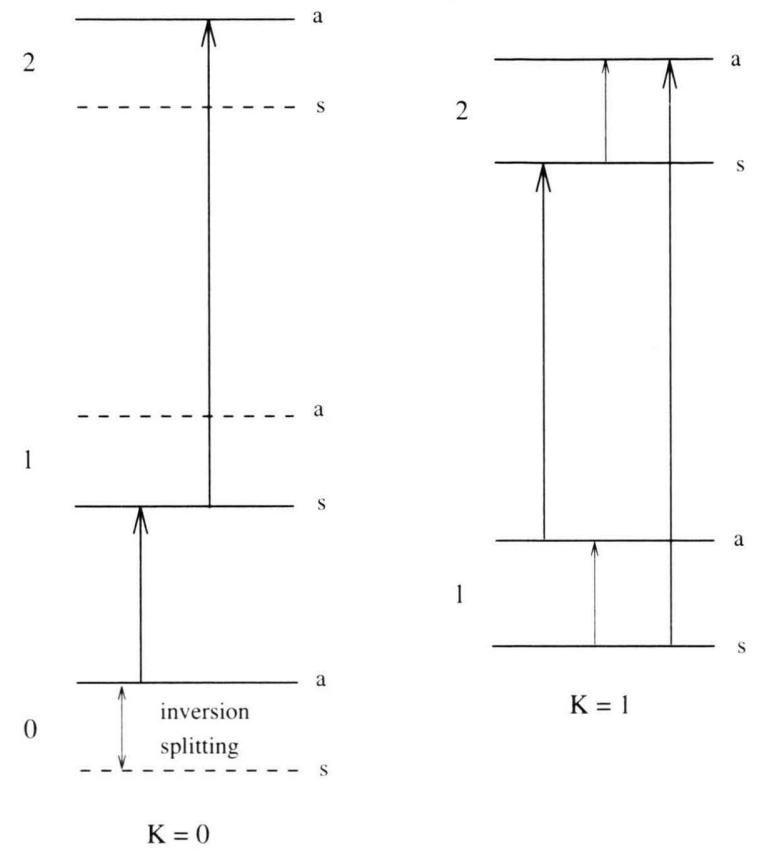

Fig. 2. Energy level diagram for the lowest ro-inversional levels of ammonia. The allowed ro-inversional and pure inversional transitions are indicated.

and their signal to noise. Since the ammonia transitions are susceptible to large pressure shifts, (i. e. about $6.1 \mathrm{MHz} /$ Torr, as measured for the $J=1-0$ transition [18]), the measurements were performed at very low pressures $(\sim 4 \mu \mathrm{bar})$ to ensure that effects of pressure shift remain negligible. In fact, the measurement errors quoted in Table 1 do not reflect the achievable accuracy. They are larger than what would be obtained from the pure measurement accuracy to allow for possible pressure effects. For well isolated and intense lines the measurement accuracy with the Cologne terahertz spectrometer operated in the Doppler limited mode is better than $\pm 5 \mathrm{kHz}$ [13]. The frequency accuracy of the system is always monitored by performing measurements on different $\mathrm{CO}$ rotational transitions.

Quite independently however, there exists an exacting test for judging the absolute accuracy of the present $\mathrm{NH}_{3}$ measurement of the $J=2-1, K=1$ transitions and their internal consistency with molecular 


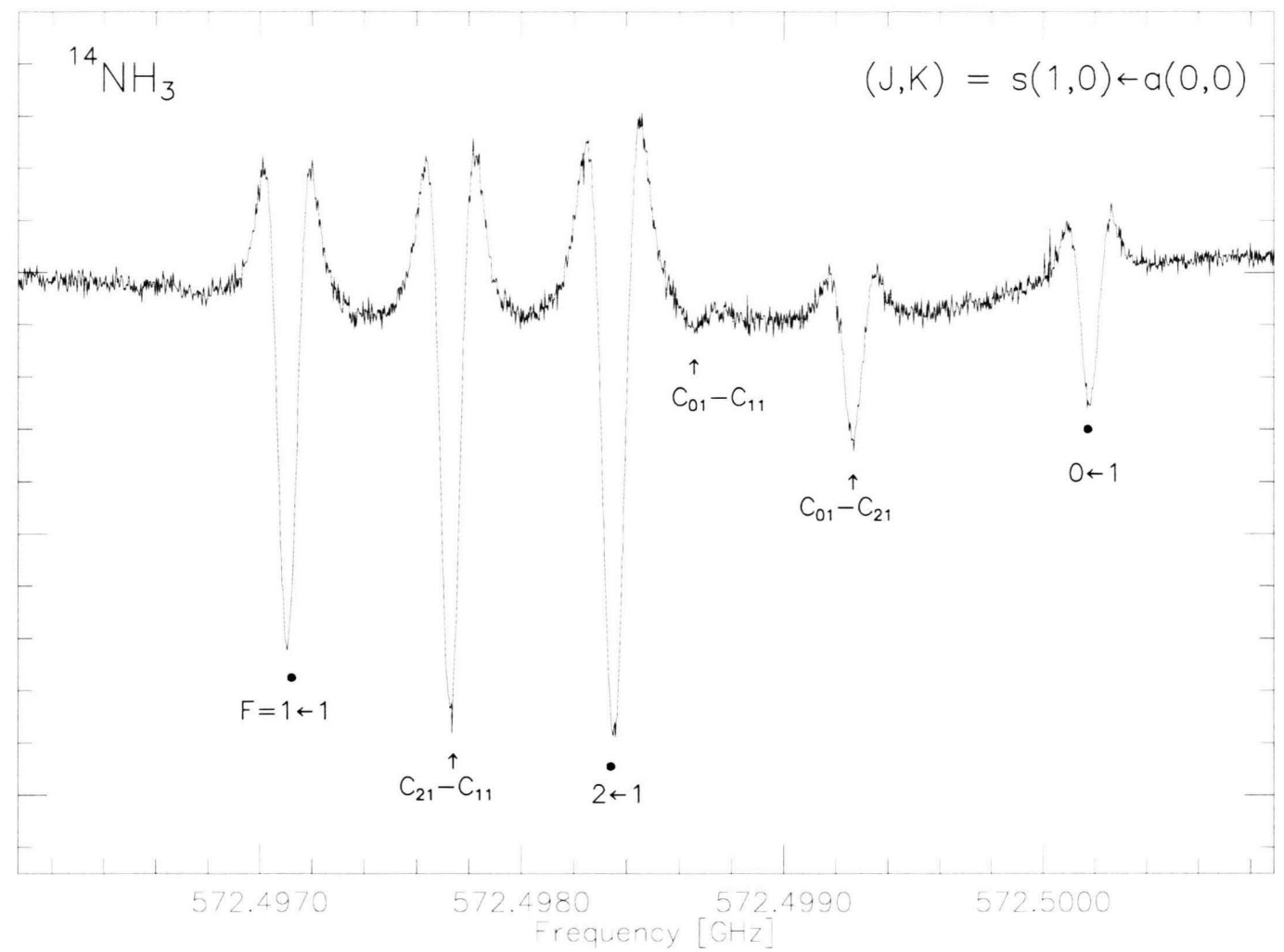

Fig. 3. Saturation-dip spectrum of the ${ }^{14} \mathrm{NH}_{3} J=1-0$ transition around $572.5 \mathrm{GHz}$. The three hyperfine components are marked by filled circles $(\bullet)$. The cross-over resonances are identified with the notation $C_{F_{1}^{\prime} F^{\prime \prime}}-C_{F_{2}^{\prime} F^{\prime \prime}}$, indicating the overlapping energy states.

beam measurements on the pure inversion transitions [19]. According to the energy level diagram for the ro-inversional levels shown in Fig. 2, the frequency difference between the two inversion split $K=1 \mathrm{com}-$ ponents of the $J=2-1$ transition has to equal the sum of the frequencies of the two inversion transitions $(1,1)$ and $(2,1)$. For ${ }^{14} \mathrm{NH}_{3}$ the frequency comparison of the various transitions agrees within $6 \mathrm{kHz}$, as will be demonstrated below. From this close coincidence, we conclude that the terahertz measurements are likely to be within the quoted $\pm 5 \mathrm{kHz}$ [13] for unblended lines although we give somewhat larger error margins to allow for possible pressure shifts. The agreement of the terahertz frequencies and the beam measurements lies within $6 \mathrm{kHz}$.

For ${ }^{15} \mathrm{NH}_{3}$ however, the agreement is not quite so good. Here only the $(1,1)$ transition has been measured with molecular beam techniques by Kukolich and Wofsy [19], whereas the $(2,1)$ transition is only known to conventional microwave accuracy [20]. In the comparison shown below, we have used the calculated $(2,1)$ transition frequency of ${ }^{15} \mathrm{NH}_{3}$ [20] rather than the measured one.

According to Table 1 and Fig. 2, the difference between the two $J=2-1$ inversion split $K=1$ terahertz lines $\Delta(K=1)=\{a(2,1)-s(1,1)\}-\{s(2,1)-$ $a(1,1)\}$ is:

$$
\begin{aligned}
& { }^{14} \mathrm{NH}_{3} \Delta(K=1)=46793.320 \mathrm{MHz}, \\
& { }^{15} \mathrm{NH}_{3} \Delta(K=1)=44669.214 \mathrm{MHz} .
\end{aligned}
$$

The frequencies of the two inversion transitions and their sum are respectively [19], [20]:

$$
\begin{array}{rlll}
{ }^{14} \mathrm{NH}_{3} & (1,1) & 23694.49548 & \mathrm{MHz}, \\
& (2,1) & 23098.81901 & \mathrm{MHz}, \\
& \text { sum } & 46793.31449 & \mathrm{MHz}, \\
{ }^{15} \mathrm{NH}_{3} & (1,1) & 22624.9311 & \mathrm{MHz}, \\
& (2,1) & 22044.26 & \mathrm{MHz}, \\
& \text { sum } & 44669.19 & \mathrm{MHz} .
\end{array}
$$


${ }^{14} \mathrm{NH}_{3}$ : Energy level diagram for the lowest ro-inversional transition $\mathbf{J}=1-()$

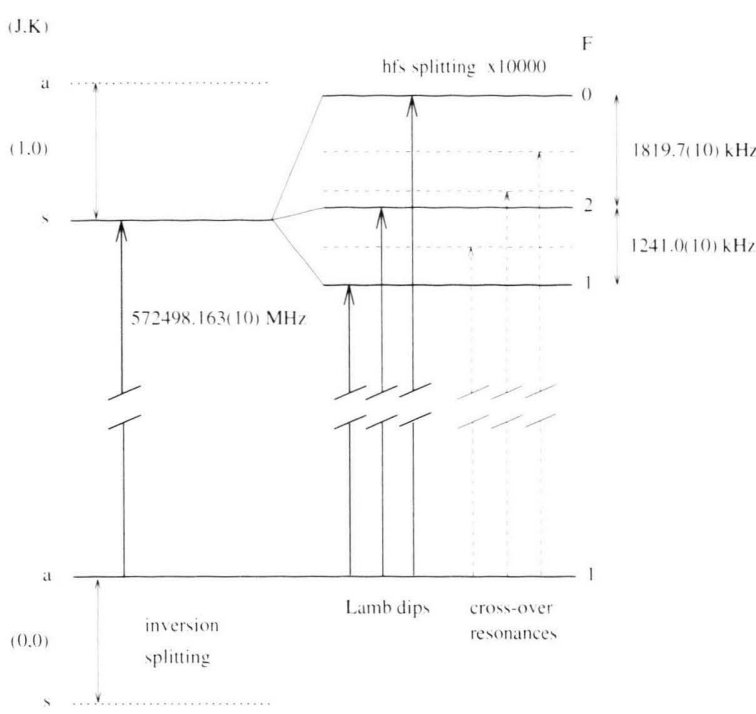

Fig. 4. Ground state energy level diagram of ammonia for the two lowest $J$ values of the $K=0$ ladder. Half of the inversion doublets is missing due to nuclear spin statistics and symmetry considerations. The hyperfine splitting for the $J=1, K=0$ s-state is indicated together with the electric dipole allowed transitions and their appropriate cross-over dips.

Thus the sum of the inversion transitions and the difference of the terhertz transitions compare as $\Delta(K=1)-\Sigma($ inv $)=0.0055 \mathrm{MHz}$ and $0.024 \mathrm{MHz}$ for ${ }^{14} \mathrm{NH}_{3}$ and ${ }^{15} \mathrm{NH}_{3}$, respectively. This proves the high degree of accuracy of the different measurements and their internal consistency. It also shows that at pressures below $4 \mu$ bar, effects of possible pressure shifts of the terahertz lines can be neglected within the measurement accuracy. The latter is assumed to be $20 \mathrm{kHz}$ for the present ammonia measurements.

\section{$J=1-0$ transition}

Recently, we succeeded in recording the $(J, K)=$ $s(1,0)-a(0,0)$ ro-inversional transition with subDoppler resolution, by operating the Cologne terahertz spectrometer in the saturation mode [21]. Since our first detection of $\mathrm{NH}_{3} J=1-0$ saturation dips, we have improved the operational conditions of the spectrometer by adjusting the $\mathrm{NH}_{3}$ pressure in the cell to fairly low values, by reducing the amplitude of the source modulation, and by ensuring the proper power
Table 2. Measured Lamp dip frequencies and cross-over resonances of ${ }^{14} \mathrm{NH}_{3}$ for the $(J, K)=s(1,0)-a(0,0)$ transition.

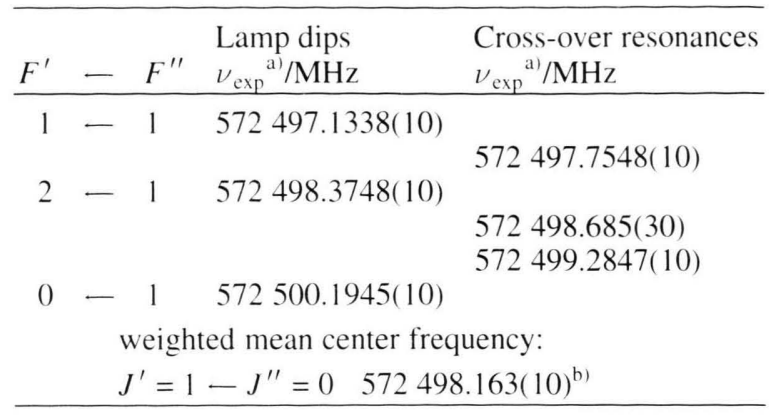

a) The quoted line positions still can contain frequency shift due to pressure effects; ${ }^{\text {b) }}$ The estimated uncertainty includes the possible contributions from the pressure shift.

level of the backward wave oscillator, BWO. The optimized Lamb dip spectrum of the ${ }^{14} \mathrm{NH}_{3} J=1-0$ transition is displayed in Figure 3. It reveals five strong absorption features and one very weak absorption: the three hyperfine components and their corresponding three cross-over dips, of which one is only faintly seen. The corresponding energy level diagram for the $J=1-0$ transition together with the appropriate crossover resonances is depicted in Figure 4. The fully resolved nuclear quadrupole hyperfine structure of the ${ }^{14} \mathrm{~N}$ nucleus displayed in this recording shows the relative intensity ratios of the hf-components close to the theoretically expected $33: 55: 11$ values, indicating that we are operating in the optically thin regime for the adjusted pressure in the $4 \mathrm{~m}$ long absorption cell. The present determination of the line positions agrees within $7 \mathrm{kHz}$ with our previous measurements quoted in reference [21], which was a first qualitative study, but are thought to be considerably more reliable. The newly measured Lamb-dip frequencies are summarized in Table 2. We also present the frequency positions of the three cross-over dips, which occur only when the transition pair has a quantum state in common and when the level splitting is comparable with the Doppler width. The details of the power spectrum of saturation dips has been worked out by Magerl et al. [22], with the result that the crossover dips occur exactly at the center of two transitions which overlap within their Doppler profile, have the same width as normal absorption dips, and their intensity is proportional to the product of the squares of the two transition dipole moments. The two strong cross-over dips in Fig. 3 arise from an overlap of each 
of the two outer hf transitions with the inner one ( $F=$ 2-1), whereas the very weak cross-over dip is caused by the bare overlap of the Doppler wings of the two outer hf-components. The measured frequencies of the cross-over dips provide a beautiful internal consistency check on the relative positions of the various spectral features to within about $1 \mathrm{kHz}$ (see Table 2). The absolute accuracy of the Lamb-dip measurements is estimated to be of the same order if pressure shifts are negligible.

The hyperfine splitting of the $J=1, K=0$ level has also been observed by Magerl et al. [22] by recording the as $\mathrm{P}(1,0)$ transition of the $\nu_{2}$ band of $\mathrm{NH}_{3}$ using sideband laser sub-Doppler spectroscopy. However our sub-Doppler measurements are two orders of magnitude more accurate. The quadrupole hfinteraction constant $e q Q=-4089.65 \mathrm{kHz}$ has been derived from various inversion transitions, after the residual $J$ and $K$ dependence has been removed [23]. Accordingly for the $J=1, K=0 \mathrm{~s}$-state, one derives $e q Q_{(J, K)}=e q Q_{(1,0)}=-4088.58 \mathrm{kHz}$. With this fixed value for the electric hf-coupling constant and the inclusion of the nitrogen nuclear spin-rotation constant $C_{\mathrm{N}}=6.7(3) \mathrm{kHz}$ normal to the symmetry axis, the observed hf-splitting is reproduced within $1 \mathrm{kHz}$. Kukolich [19] obtained $C_{\mathrm{N}}=6.80734 \mathrm{kHz}$.

\section{Interstellar $\mathrm{NH}_{3}$ : ro-vibrational transitions}

$\mathrm{NH}_{3}$ is an ubiquitous interstellar molecule with high abundance. The ro-vibrational transitions are especially valuable because they require high density gas to excite them and thus they probe the cores of star formation regions. In addition they allow us to sample the $K=0$ levels, which do not have inversion lines, a fact which is particularly important for the $(J, K)=$ $(0,0)$ ground state. Many high excitation metastable (e. g. $(J, K)=(14,14))$ and non-metastable $(11,10)$ pure inversion transitions [24] have been used to probe the temperature and density structures of molecular clouds [25], suggesting that the ro-inversional transitions are excited under interstellar conditions in areas of only small solid angle. The high excitation nonmetastable $\mathrm{NH}_{3}$ emission probably comes mainly from very compact regions of warm and dense gas in molecular cloud cores. Interstellar emission of pure ro-inversion transitions has been detected towards the center of the Orion-A nebula by using the Kuiper Airborne Observatory, KAO. Different ro-inversion tran- sitions seem to be emitted from different excitation regions. The $J=1-0$ transition at $572.5 \mathrm{GHz}$ has been observed in Orion A, with the emission originating from the quiescent molecular cloud material known as the "spike" component [26]. The emission of the $J$ $=4-3, K=3$ transition at $124.6474 \mu \mathrm{m}$ seems to arise from the "hot-core" gas rather than from the "spike" region, since only the "hot-core" component provides sufficient density (e.g. $n>10^{7} \mathrm{~cm}^{-3}$ ) for ammonia molecules to produce the observed emission intensity [27]. The submillimeter and far IR ro-inversional transitions of ammonia happen to occur at wavelengths where the Earth's atmosphere is quite opaque, and consequently they can not be observed from ground based telescopes.

\section{Conclusion}

For the first time, the $J=2-1$ ground state $\mathrm{R}$ branch transitions of ammonia have been measured with microwave accuracy. The new data allow us to use Ritz' combination principle for an independent check on the accuracy of the terahertz measurements (see Fig. 2): the sum of the frequency of the lower $K=1$ component of the $J=2-1$ transition and the frequencies of the two involved pure inversional transitions $\nu(1,1)+\nu(2,1)$ has to equal the frequency of the upper $K=1$ component of the $J=2-1$ transition. For ${ }^{14} \mathrm{NH}_{3}$ their agreement is within $6 \mathrm{kHz}$.

We have also presented Lamb-dip spectra for the ground state $J=1-0, K=0$ transition, the hf-structure of which has been completely resolved and measured to an accuracy of $1 \mathrm{kHz}$. The appropriate three crossover dips have been recorded as well.

The ro-inversional transitions of $\mathrm{NH}_{3}$ are of astrophysical importance in probing both high and low density interstellar gas, in emission and absorption respectively. These observations are impossible because of atmospheric extinction at ground based observatories. Interfering telluric absorption lines cause difficulties even at high altitudes attained by the NASA$\mathrm{KAO}$, with which the $J=1-0$ and the $J=4-3, K=3$ lines have been detected in emission [26], [27]. Free of telluric interference will be the $\mathrm{NH}_{3}$ ro-inversional transitions scheduled for observation with the recently launched European Infrared Space Observatory, ISO.

Inversionally excited $\mathrm{NH}_{3}$ is also of astrophysical interest. We are presently investigating its terahertz spectrum. During the course of this work, we have 
recorded the Lamb-dip spectra of the $J=1-0$ transition in the excited vibrational state $v_{2}=1$ and resolved its hyperfine structure.

\section{Acknowledgements}

This work has been supported in part by the Deutsche Forschungsgemeinschaft (DFG) via special research grant SFB 301 and Wi 391/7-1, as well as by the Ministry of Science and Technology of the State Nordrhein-Westfalen. The work of S. P. B. at Cologne was made possible by the DFG through grants aimed to support Eastern and Central European countries and the republics of the former Soviet

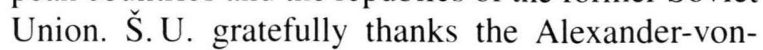
Humboldt Stiftung for granting an extension of his fellowship.
[1] C. E. Cleeton and N. H. Williams, Phys. Rev. 45, 234 (1934).

[2] J. P. Gordon, H. J. Zeiger, and C. H. Townes, Phys. Rev. 99, 59 (1955).

[3] A. C. Cheung, D. M. Rank, C. H. Townes, D. D. Thornton, and J. Welch, Phys. Rev. Letters 21, 1701 (1968).

[4] D. M. Dennison and G. E. Uhlenbeck, Phys. Rev. 41, 313 (1932).

[5] C. C. Costain, Phys. Rev. 82, 108 (1951).

[6] C. H. Townes and A. L. Schawlow, Microwave Spectroscopy, McGraw-Hill, New York 1955.

[7] W. Gordy and R. L. Cook Microwave Molecular Spectra, J. Wiley \& Sons, New York 1984.

[8] P. Helminger and W. Gordy, Bull. Amer. Phys. Soc. 12, 543 (1967), also Phys. Rev. 188, 100 (1971).

[9] P. Helminger, F. C. DeLucia, and W. Gordy, J. Mol. Spectrosc. 39, 94 (1971).

[10] A. F. Krupnov, L.I. Gershtein, V. G. Shustrov, and V.V. Polyakov, Izv. VUZ. Radiofizika 12, 1584 (1969).

[11] Š. Urban, V. Špirko, D. Papoušek, J. Kauppinen, S. P. Belov, L. I. Gershstein, and A. F. Krupnov, J. Mol. Spectrosc. 88, 274 (1981).

[12] Š. Urban, R. D’Cunha, K. N. Rao, and D. Papoušek, Can. J. Phys. 62, 1775 (1984).

[13] S. P. Belov, F. Lewen, Th. Klaus, and G. Winnewisser, J. Mol. Spectrosc. 174, 606 (1995).

[14] G. Winnewisser, Vibrational Spectroscopy 8, 241 (1995).
[15] F. Lewen, S. P. Belov, F. Maiwald, Th. Klaus, and G. Winnewisser, Z. Naturforsch. 50a, 1 (1995).

[16] W. Etzenbach, A. H. Saleck, M. Liedtke, and G. Winnewisser, Can. J. Phys. 72, 1315 (1994).

[17] Š. Urban, S. Klee, and K. M. T. Yamada, J. Mol. Spectrosc. 168, 384 (1994).

[18] S. P. Belov, V. P. Kazakov, A. F. Krupnov, V. N. Markov, A.A. Mel'nikov, V.A. Skvortsov, and M. Yu. Tretyakov, J. Mol. Spectrosc. 94, 264 (1982).

[19] S. G. Kukolich, Phys. Rev. 156, 83 (1967), also S. G. Kukolich and S. C. Wofsy, J. Chem. Phys. 52, 5477 (1970).

[20] H. Sasada, J. Mol. Spectrosc. 83, 15 (1980).

[21] S. P. Belov, Th. Klaus, G. M. Plummer, R. Schieder, and G. Winnewisser, Z. Naturforsch. 50 a, 1187 (1995).

[22] G. Magerl, W. Schupita, J. M. Frye, W. A. Kreiner, and T. Oka, J. Mol. Spectros. 107, 72 (1984).

[23] J. T. Hougen, J. Chem. Phys. 37, 4207 (1972).

[24] see e. g. S. Hüttemeister, T. L. Wilson et al., Astron. \& Astrophys. 294, 667 (1995)., ibid 276, 445 (1993).

[25] P. T. P. Ho and C. H. Townes, Ann. Rev. Astron. Astrophys. 21, 239 (1983).

[26] J. Keene, G. A. Blake, and T. G. Phillips, Astrophys. J. 271, L27 (1983).

[27] C. H. Townes, R. Genzel, D. M. Watson, and J. W. V. Storey, Astrophys. J. 269, L11 (1983). 